\title{
Introducción a los estudios de caso de eventos dañinos en La Paz, Lima y Quito
}

Introduction aux études de cas d'évènements à l'origine de dommages à La Paz, Lima et Quito

Introduction to case studies of events resulting in damages in La Paz, Lima and Quito

Robert D'Ercole, Sébastien Hardy y Jérémy Robert

\section{(2) OpenEdition \\ Journals}

\section{Edición electrónica}

URL: http://journals.openedition.org/bifea/2253

DOI: $10.4000 /$ bifea.2253

ISSN: 2076-5827

Editor

Institut Français d'Études Andines

\section{Edición impresa}

Fecha de publicación: 1 diciembre 2009

Paginación: 469-474

ISSN: 0303-7495

Referencia electrónica

Robert D'Ercole, Sébastien Hardy y Jérémy Robert, «Introducción a los estudios de caso de eventos dañinos en La Paz, Lima y Quito », Bulletin de I'Institut français d'études andines [En línea], 38 (3) | 2009, Publicado el 01 junio 2010, consultado el 17 noviembre 2020. URL : http://journals.openedition.org/ bifea/2253 ; DOI : https://doi.org/10.4000/bifea.2253

\section{BY NO ND}

Les contenus du Bulletin de l'Institut français d'études andines sont mis à disposition selon les termes de la licence Creative Commons Attribution - Pas d'Utilisation Commerciale - Pas de Modification 4.0 International. 


\section{Parte 1}

\section{LECCIONES DEL PASADO}

2. De los eVentos del ayer a la VULNerabiLIDAD DE HOY

Des ÉVÈnEMENTS D'HIER À LA VULNÉRABILITÉ D'AUJOURD'HUI

From YesteRDAY'S EVENTS TO TODAY'S VULNERABILITY 



\title{
Introducción a los estudios de caso de eventos dañinos en La Paz, Lima y Quito
}

\author{
Robert D'Ercole* \\ Sébastien Hardy** \\ Jérémy Robert***
}

\section{Resumen}

Los eventos de ayer permiten comprender la vulnerabilidad de hoy. En concordancia con esta idea, se presentan siete estudios de caso correspondientes a accidentes y desastres ocurridos en La Paz, Lima y Quito desde 1987. En esta introducción se exponen los criterios considerados para su selección. Se ha adoptado una metodología común de análisis para poder hacer comparaciones entre ciudades y extraer un máximo de enseñanzas de estos eventos. Esta metodología toma en cuenta el contexto del evento, sus consecuencias, las vulnerabilidades estructurales, las vulnerabilidades ligadas al manejo de crisis y las incidencias en materia de política de reducción de riesgos.

Palabras clave: estudios de caso, análisis retrospectivos, accidentes y desastres, crisis, ciudades, criterios de selección, metodología

\section{Introduction aux études de cas d'évènements à l'origine de dommages à La Paz, Lima et Quito}

\section{Résumé}

Les évènements d'hier permettent de comprendre la vulnérabilité d'aujourd'hui. Suivant cette idée, sept études de cas sont présentées, correspondant à des accidents et des catastrophes survenus à

* Institut de Recherche pour le Développement (IRD), UR 029, programa Pacivur, Calle Teruel 357, Miraflores, Casilla 18-1209, Lima 18, Perú. E-mail: robert.dercole@ird.fr

** Institut de Recherche pour le Développement (IRD), UR 029, programa Pacivur, Av. Hernando Siles 5290 esq. calle 7, CP 9214 Obrajes, La Paz, Bolivia. E-mail: sebastien.hardy@ird.fr

*** Institut Français d'Études Andines (IFEA)-Université de Savoie, CISM, Laboratorio EDYTEM (CNRS). E-mail: Jeremy.Robert@univ-savoie.fr 
La Paz, Lima et Quito depuis 1987. Dans cette introduction, les critères ayant guidé ce choix sont expliqués. Afin de permettre la comparaison entre villes et de tirer le maximum d'enseignements de ces évènements, une méthodologie commune d'analyse a été adoptée. Elle prend en compte le contexte de l'évènement, ses conséquences, les vulnérabilités structurelles, les vulnérabilités liées à la gestion de crise et les effets en matière de politique de réduction des risques.

Mots clés : études de cas, analyses rétrospectives, accidents et catastrophes, crise, villes, critères de sélection, méthodologie

\title{
Introduction to case studies of events resulting in damages in La Paz, Lima and Quito
}

\begin{abstract}
Yesterday's events allow to understanding today's vulnerability. Within this scope, seven case studies are presented based on accidents and disasters that have occurred in La Paz, Lima and Quito since 1987. The introduction presents the criteria set for their selection. In order to be able to compare cities and learn as much as possible from these events, a common analysis methodology has been adopted. It focuses on the event's context, its consequences, the structural vulnerabilities, the vulnerabilities linked to the crisis management and the impacts on the risk reduction policy.
\end{abstract}

Key Words: case studies, retrospective analysis, accidents and disasters, crisis, cities, selection criteria, methodology

El conocimiento de los eventos que originan daños y perturbaciones permite ingresar directamente al análisis de la vulnerabilidad de los territorios urbanos. Numerosos autores subrayan las ventajas de los análisis retrospectivos (Léone \& Velásquez, 1996; Metzger \& Peltre, 1996; Pigeon, 1995). En este contexto, el examen meticuloso de las bases de datos que catalogan los accidentes y desastres ocurridos en las capitales andinas es mucho más rico en enseñanzas que lo que anuncia a primera vista la larga y monótona enumeración de eventos. Un primer análisis permite evidenciar la frecuencia y la recurrencia de estos eventos. Su profundización desemboca en la caracterización de los daños en el transcurso del tiempo y, cuando la base de datos lo permite, en su espacialización1. Este análisis de eventos constituye una primera etapa en la aprehensión de la vulnerabilidad de los territorios, pero no es suficiente. Es importante salir de un esquema estadístico y cartográfico y penetrar en el corazón mismo del evento para conocer mejor sus causas y su alcance. En efecto, la vulnerabilidad se edifica sobre el largo plazo y solo un análisis profundo de los eventos permite comprender los procesos de su construcción.

1 Ver en este volumen el artículo de R. D'Ercole, S. Hardy y J. Robert, pp. 433-465. 
El análisis retrospectivos de los eventos permite comprender las condiciones que han favorecido el daño, diferenciando la vulnerabilidad de fondo de las condiciones más contingentes al evento en sí. Como tratan de situaciones reales, estos estudios de caso son muy útiles: consideran una vulnerabilidad que ya no es virtual y llevan a buscar soluciones para reducirla. Por esta razón, tales análisis —-más allá del interés en reconstruir a posteriori un evento que ha causado daños para conocerlo mejor: es decir, en una temporalidad que ya no es la de la emergencia-constituyen, junto con el enfoque estadístico y cartográfico, una de las primeras etapas metodológicas de análisis de la vulnerabilidad en medios urbanos.

Estos análisis son bastante fáciles de efectuar. No requieren recursos financieros o humanos importantes, y ofrecen rápidamente resultados satisfactorios que permiten orientar las políticas de reducción de riesgos. En consecuencia, son un método eficaz de producción de conocimientos, en particular para las ciudades de los países en desarrollo que no disponen de presupuestos importantes para la actividad preventiva. Estos análisis retrospectivos son también de innegable utilidad para el investigador. Además de la identificación de vulnerabilidades y la puesta en evidencia de su carácter particular o recurrente, lo que permite la comparación, se puede efectuar una reflexión sobre la manera con la cual se articulan estas vulnerabilidades para producir unas crisis más o menos graves. Por último, el ejercicio resulta tanto más pertinente por cuanto estos eventos, en particular los que generan daños importantes y/o fuertemente mediatizados, originan a menudo cambios en las políticas de gestión del riesgo. Es importante captar estas evoluciones institucionales, generalmente sinónimas de reducción de la vulnerabilidad: es lo que permite el análisis retrospectivo.

\section{SELECCIÓN DE LOS EVENTOS}

En este contexto, hemos procedido al análisis de algunos de los eventos pasados, accidentes y desastres, que han afectado La Paz, Lima y Quito. Los fenómenos de origen natural, antrópico o mixto que han provocado estos eventos, causaron daños significativos, de manera directa e indirecta. En su conjunto son eventos recientes, dada la poca información disponible para eventos antiguos. Más allá de estos puntos comunes, los eventos han sido seleccionados siguiendo cierto número de criterios que permiten abordar el tema de la vulnerabilidad de la manera más amplia posible.

En primer lugar, se han escogido eventos ligados a fenómenos de diferente tipo (accidentes tecnológicos, inundaciones, huaycos - $-\mathrm{O}$ aluviones—, erupciones volcánicas, etc.). Al mismo tiempo, la idea es obtener una muestra representativa de los eventos más habituales y/o más característicos de la ciudad. Las granizadas e inundaciones constituyen fenómenos representativos de los eventos dañinos que afectan regularmente a La Paz, aunque su impacto sea generalmente menor que aquellos ocurridos el 19 de febrero de 2002 (seleccionados aquí). La erupción del Guagua Pichincha en 1999 es otro ejemplo. A escala humana, las erupciones no son frecuentes en Quito; sin embargo, sí son características de la 
capital ecuatoriana, dada la historia geológica de los terrenos sobre los cuales está asentada y en la medida en que está rodeada de varios volcanes activos.

Nos ha parecido importante también tratar de eventos que, al inicio de la crisis, han afectado, progresiva o súbitamente, espacios más o menos extensos. A pesar de su carácter espectacular, el incendio de Santa Rosa de Chillogallo, ocurrido en abril de 2003, afectó un espacio muy limitado de la ciudad de Quito. Fue provocado por la perforación delictuosa de un poliducto ocasionada por un robo de hidrocarburos y afectó un espacio muy preciso de la ciudad. El evento fue, en consecuencia, súbito y muy localizado. La erupción del volcán Pichincha en Quito fue, al contrario, un evento «anunciado» para los quiteños, por la puesta en alerta, los medios de comunicación y los signos precursores (sismos y actividad freática), viéndose afectada gran parte del distrito metropolitano.

Hemos seleccionado igualmente eventos que han tenido efectos territoriales directos o indirectos más o menos extensos. Aquí no se trata ya de lugares directamente afectados por tal o cual fenómeno, sino de espacios situados a distancia donde se han sentido consecuencias del evento. La diferencia entre estos dos tipos de espacios es a veces muy importante. Por ejemplo, en 2008, el hundimiento de El Trébol en Quito fue un fenómeno físico muy localizado en el espacio, cuyos efectos, particularmente en términos de movilidad de las personas y de los bienes, alcanzaron gran parte del territorio metropolitano. También es el caso de la ruptura de la canalización de agua de Hampaturi en febrero de 2008, cuyos efectos directos se registraron por lo menos sobre un tercio del territorio de la aglomeración de La Paz. Estos ejemplos demuestran que el lugar de ocurrencia del evento no es sinónimo de espacio dañado y que estos dos espacios son a menudo muy diferentes, e incluso distantes uno de otro.

Algunos eventos han sido escogidos por su antigüedad relativa, con el propósito de estudiar sus incidencias sobre un plazo más largo. Dos de ellos tienen 10 o más años: la alerta y la erupción del Pichincha en Quito (1998-1999) y los huaycos de Lurigancho-Chosica, Lima, ocurridos en 1987. La retrospectiva en relación con el análisis del evento autoriza el examen de las medidas de reducción de riesgos tomadas por los gestores urbanos, y sus efectos. En otras palabras, permite captar la capacidad de reacción de la sociedad urbana frente al evento.

Por último, hemos seleccionado un evento ligado a un fenómeno característico del Perú: el terremoto. El lugar escogido no es, sin embargo, aquel donde los daños fueron más importantes. En efecto, el estudio de caso atañe a la MIRR (Margen Izquierda del Río Rímac, Cercado de Lima), sector marginal de Lima situado a 200 km del epicentro del sismo del 15 de agosto de 2007 que afectó severamente las provincias de Pisco, Chincha e Ica. El ejercicio resultó interesante, porque trata de un conjunto de barrios donde los efectos del sismo, aun cuando no alcanzan la magnitud de aquellos observados a proximidad del epicentro, han sido subestimados y relativizados tanto por la población como por las autoridades municipales. Esta situación revela profundas vulnerabilidades que han marcado el manejo de crisis en la MIRR. 


\section{METODOLOGÍA}

Nuestro objetivo radica en extraer el máximo de enseñanzas de los análisis retrospectivos de eventos que han originado daños en las tres capitales. Para ello, pero también dentro de una perspectiva comparativa entre La Paz, Lima y Quito, hemos tratado de seguir una misma metodología en cada uno de estos análisis.

Primero el evento es reconstituido de manera sucinta, con la presentación del contexto en el cual apareció y una rápida descripción de los hechos y las características del fenómeno perturbador y/o destructor. Enseguida se exponen sus consecuencias directas (daños a las personas, a los bienes y a las actividades), tal como pueden aparecer en las bases de datos, los documentos de archivo o la prensa. También se presentan las consecuencias indirectas, las mismas que han sido objeto de investigaciones específicas (revistas de prensa, entrevistas), en la medida en que no aparecen prácticamente nunca en las bases de datos.

En un segundo momento, tratamos de tomar distancia respecto de las informaciones, frecuentemente muy factuales, para reflexionar sobre las vulnerabilidades estructurales (o de fondo) que originan el evento y la importancia de los daños. Por lo tanto, el análisis de los factores de vulnerabilidad ha requerido penetrar en el corazón del evento para extraer mecanismos técnicos, sociales, socioeconómicos o institucionales, mucho más complejos que lo que el evento mismo pudo sugerir en un primer momento. Los datos obtenidos han sido cruzados entre sí para comprender mejor el proceso de construcción de la vulnerabilidad. Por ejemplo, los datos factuales sobre la ruptura de la canalización de agua en Hampaturi han sido cruzados con los de la evolución de la situación de la empresa concesionaria del servicio de producción y de abastecimiento en agua potable de La Paz. Este cruce no es evidente $y$, sin embargo, aclara de manera muy útil la interpretación del evento y de los daños.

El énfasis ha sido puesto enseguida sobre el manejo de las crisis desencadenadas por estos eventos. El propósito fue evaluar las capacidades de gestión de situación de crisis de los diferentes actores, en las diferentes capitales, en función de los tipos de eventos, e identificar las principales dificultades encontradas. Las temporalidades (por ejemplo el hecho de que un evento se produzca de día o de noche) también han sido consideradas. Se pudieron identificar entonces vulnerabilidades más específicas del manejo de crisis, complementarias de las vulnerabilidades estructurales.

Los estudios de caso concluyen con el análisis de las consecuencias de cada crisis a más o menos largo plazo, según la perspectiva disponible; particularmente las incidencias en materia de aprendizaje, de adaptación y de políticas de gestión del riesgo.

Siguiendo esta progresión metodológica, los análisis retrospectivos de los eventos elegidos ofrecen información útil, tanto para el investigador como para el planificador o el gestor de las crisis. Esta sección concluye, entonces, con una síntesis destinada a extraer enseñanzas de estos estudios de caso. 


\section{Referencias citadas}

LÉONE, F. \& VELÁSQUEZ, E., 1996 - Analyse en retour de la catastrophe de La Josefina (Équateur, 1993) : contribution à la connaissance du concept de vulnérabilité appliqué aux mouvements de terrain. Bulletin de I'Institut Français d'Études Andines, 25 (3): 461-478.

METZGER, P. \& PELTRE, P., 1996 - Gestion de l'environnement urbain et risques «naturels». La problématique des quebradas à Quito (Équateur). Bulletin de I'Institut Français d'Études Andines, 25 (3): 531-552.

PIGEON, P., 2005 - Géographie critique des risques, 217 pp.; París: Economica. 